\title{
SUR UN PROBLÈME PARABOLIQUE-ELLIPTIQUE
}

\author{
Philippe Benilan $^{1}$ And Petra Wittbold ${ }^{2}$
}

\begin{abstract}
We prove existence (uniqueness is easy) of a weak solution to a boundary value problem for an equation like $(v-1)_{t}^{+}=v_{x x}+F(v)_{x}$ where the function $F: \mathbb{R} \rightarrow \mathbb{R}$ is only supposed to be locally lipschitz continuous. In order to replace the lack of compactness in $t$ on $v<1$, we use nonlinear semigroup theory.

Résumé. Nous prouvons l'existence (l'unicité est facile) d'une solution faible pour un problème aux limites associé à une équation du type $(v-1)_{t}^{+}=v_{x x}+F(v)_{x}$ où la fonction $F: \mathbb{R} \rightarrow \mathbb{R}$ est seulement supposée localement lipschitzienne. Pour pallier au manque de compacité en $t$ lorsque $v<1$, nous utilisons la théorie des semi-groupes non-linéaires.
\end{abstract}

AMS Subject Classification. 35K60, 35K65, 47H06, 47H20.

Reçu : 20 Novembre 1997.

\section{INTRODUCTION}

De très nombreux travaux sont consacrés à l'existence de solutions faibles pour des problèmes aux limites associés à des équations paraboliques-elliptiques du type

$$
b(v)_{t}=\operatorname{div}_{x} a(v, D v)
$$

où $b: \mathbb{R} \rightarrow \mathbb{R}$ est continue croissante et $a:(k, \xi) \in \mathbb{R} \times \mathbb{R}^{N} \rightarrow \mathbb{R}^{N}$ est continue en $k$ et monotone coercive en $\xi$.

Citons en particulier les travaux de Alt et Luckhaus [1], où à coté d'hypothèses permettant d'utiliser un bon cadre variationnel, apparait clairement la condition suivante :

$$
a(k, \xi)=\underline{\mathbf{a}}(b(k), \xi) \text { avec } \underline{\mathbf{a}}: \mathcal{R}(b) \times \mathbb{R}^{N} \rightarrow \mathbb{R}^{N} \text { continue. }
$$

Si $b(k)$ est strictement croissante, cette condition est automatiquement satisfaite et donc superflue ; mais sinon elle parait essentielle pour obtenir des solutions faibles pour l'équation (1) : en effet, heuristiquement, dans un procédé d'approximation, on obtiendra une compacité (forte) de $b(v)$ dans la variable $t$, et de $v$ dans la variable d'espace $x$; compte-tenu de la continuité de $b(k)$, on aura donc une compacité de $b(v)$ en $(t, x)$; sous l'hypothèse (2), on pourra alors, avec un argument de pseudo-monotonie pour le gradient $D v$, passer à la limite ; par contre, sans cette hypothèse, on manquera d'une compacité de $v$ en temps pour passer à la limite dans $a(v, D v)$.

Cette situation apparait même dans un exemple aussi simple que l'équation en une dimension d'espace

$$
(v-1)^{+}{ }_{t}=v_{x x}+v^{2}{ }_{x} .
$$

\footnotetext{
${ }^{1}$ Equipe de Mathématiques, UMR CNRS 6623, Université de Franche-Comté, 25030 Besançon Cedex, France. e-mail: benilan@math.univ-fcomte.fr

${ }^{2}$ UFR de Mathématiques et Informatique, 7 rue René Descartes, 67084 Strasbourg Cedex, France. e-mail: wittbold@math.u-strasbg.fr
} 
Dans [3], nous avons montré que, sous des hypothèses très générales, on pouvait toujours, sans la condition (2), développer l'approche par la théorie des semi-groupes non linéaires, du problème aux limites associé à (1) sur $(0, \infty) \times \Omega$, avec des conditions de Dirichlet $v=0$ sur $(0, \infty) \times \partial \Omega$; nous obtenions ainsi, sans la condition $(2)$, l'existence de "bonnes solutions" au sens de la théorie des semi-groupes non linéaires ; nous avons aussi montré que sous la condition (2), les "bonnes solutions" étaient solutions faibles de (1). Et nous posions la question : cette condition est-elle essentielle comme elle parait l'être ? en particulier dans l'exemple de l'équation (3), y a-t-il existence de solutions faibles ?

Dans cet article, nous montrons qu'il y a effectivement existence (et unicité) de solutions faibles pour le problème aux limites de Cauchy-Dirichlet associé à (3). Nous considérerons, plus généralement que (3), une équation

$$
b(v)_{t}=v_{x x}+F(v)_{x}
$$

où $F: \mathbb{R} \rightarrow \mathbb{R}$ est localement lipschitzienne, et la fonction continue croissante $b(k)$ vérifie :

Il existe une partie fermée négligeable $K$ de $\mathbb{R}$ telle que

$b$ soit continuement dérivable sur $U=b^{-1}(\mathbb{R} \backslash K)$

avec $b^{\prime}(k)>0$ pour tout $k \in U$.

Il est clair que cette condition (5) est en particulier vérifiée si les "plats" de la fonction $b$, c'est à dire les valeurs $l \in \mathbb{R}$ telles que $b^{-1}(l)$ ait un intérieur non vide, sont isolés, et $b$ est continuement dérivable de dérivée strictement positive sur tout intervalle ouvert sur lequel elle est strictement croissante ; cette condition est évidemment vérifiée par la fonction $b(k)=(k-1)^{+}$considérée dans $(3)$.

Nous allons prouver le résultat suivant pour le problème aux limites de Cauchy-Dirichlet associé à (4) sur $(0, \infty) \times(0,1):$

Théorème. Soient $F: \mathbb{R} \rightarrow \mathbb{R}$ localement lipschitzienne et $b: \mathbb{R} \rightarrow \mathbb{R}$ continue croissante vérifiant (5). Pour tout $v_{0} \in L^{\infty}(0,1)$, il existe une unique solution (faible) $v$ du problème :

$$
\begin{aligned}
& v \in L^{\infty}((0, \infty) \times(0,1)) \cap L^{2}\left(0, \infty ; W_{0}^{1,2}(0,1)\right) \\
& \text { et pour tout } \zeta \in \mathcal{D}([0, \infty[\times] 0,1[) \text {, on } a \\
& \iint\left(b(v) \zeta_{t}+v \zeta_{x x}-F(v) \zeta_{x}\right) d t d x+\int b\left(v_{0}\right) \zeta(0, \cdot) d x=0 .
\end{aligned}
$$

De plus

$$
-\left\|v_{0}^{-}\right\|_{\infty} \leq v \leq\left\|v_{0}^{+}\right\|_{\infty} \text { p.p. } \operatorname{sur}(0, \infty) \times(0,1)
$$

et si $\underline{v}$ est la solution correspondante à une autre donnée initiale $v_{0}$, alors

$$
\int(b(v)(t, \cdot)-b(\underline{v})(t, \cdot))^{+} d x \leq \int\left(b\left(v_{0}\right)-b\left(\underline{v}_{0}\right)\right)^{+} d x \quad \text { pour tout } t \geq 0
$$

et

$$
b\left(v_{0}\right) \leq b\left(\underline{v}_{0}\right) \quad \Rightarrow \quad v \leq \underline{v} \quad \operatorname{sur}(0, \infty) \times(0,1) .
$$

La section 2 sera consacrée à la preuve de ce Théorème. Dans la section 3, nous ferons quelques remarques sur la généralité de ce résultat. 


\section{Preuve DU THÉORÈME}

On peut toujours supposer

$$
F(0)=0, b(0)=0
$$

On a le résultat élémentaire suivant :

Lemme 1. Étant donné $f \in L^{1}(0,1)$, il existe un unique $v \in W^{2,1}(0,1) \cap W_{0}^{1,1}(0,1)$ tel que $-\left(v^{\prime \prime}+F(v)^{\prime}\right)=f$ p.p. sur $(0,1)$. De plus l'application $f \rightarrow v$ est croissante, continue de $L^{1}(0,1)$ dans $W^{2,1}(0,1)$, et lipschitzienne de $L^{1}(0,1)$ dans $\mathcal{C}^{1}([0,1])$ sur les bornées de $L^{1}(0,1)$.

Preuve. Pour être complet, indiquons les estimations pour le problème considéré. Étant donné $v \in W^{2,1}(0,1) \cap$ $W_{0}^{1,1}(0,1)$ et $f=-\left(v^{\prime \prime}+F(v)^{\prime}\right)$, on a

$$
4\|v\|_{\infty}^{2} \leq\left\|v^{\prime}\right\|_{2}^{2}=\int f v \leq\|f\|_{1}\|v\|_{\infty}
$$

et donc

$$
\|v\|_{\infty} \leq \frac{1}{4}\|f\|_{1}
$$

Considérons pour $i=1,2, v_{i} \in W^{2,1}(0,1) \cap W_{0}^{1,1}(0,1)$ et $f_{i}=-\left(v_{i}^{\prime \prime}+F\left(v_{i}\right)^{\prime}\right)$; posons $v=v_{1}-v_{2}, f=f_{1}-f_{2}$ et $g=\chi_{\left\{v_{1} \neq v_{2}\right\}}\left(\left(F\left(v_{1}\right)-F\left(v_{2}\right)\right) /\left(v_{1}-v_{2}\right) ;\right.$ on a $g \in L^{\infty}(0,1)$ et, compte-tenu de (11),

$$
C:=\|g\|_{\infty} \leq\left\|F^{\prime}\right\|_{L^{\infty}(-R, R)} \quad \text { avec } R=\frac{1}{4} \max \left(\left\|f_{1}\right\|_{1},\left\|f_{2}\right\|_{1}\right) ;
$$

d'autre part $v \in W^{2,1}(0,1) \cap W_{0}^{1,1}(0,1), v^{\prime}+g v \in W^{1,1}(0,1)$ et $-\left(v^{\prime}+g v\right)^{\prime}=f$ p.p. sur $(0,1)$. On en déduit $\left\|v^{\prime}+g v\right\|_{\infty} \leq(1 / 2)\|f\|_{1}$, d'où

$$
\left\|\left(v_{1}-v_{2}\right)^{\prime}\right\|_{\infty} \leq(1 / 2) e^{C / 2}\left\|f_{1}-f_{2}\right\|_{1}
$$

Enfin si $f \leq 0$, alors $v^{\prime}+g v$ est croissante sur $[0,1]$, et donc

$$
f_{1} \leq f_{2} \quad \Rightarrow \quad v_{1} \leq v_{2}
$$

Le lemme est alors immédiat.

D'après le Corollary 4.5 de [3] si $v$ et $\underline{v}$ sont solutions de (6) associées à $v_{0}$ et $\underline{v}_{0}$, alors on a (8); en particulier si $v_{0}=\underline{v}_{0}$, alors $b(v)=b(\underline{v})$ et donc d'après l'équation, $(v-\underline{v})_{x x}+(F(v)-F(\underline{v}))_{x}=0$ dans $\mathcal{D}^{\prime}(] 0, \infty[\times] 0,1[)$; de la même manière que dans la preuve du Lemme 1 , ceci implique immédiatement $v=\underline{v}$. On a donc montré l'unicité d'une solution $v$ de (6) et la propriété (8) ; il reste donc à prouver l'existence d'une solution et les propriétés (7) et (9).

Notons d'abord que si $v \in L_{l o c}^{\infty}\left(\left[0, \infty[\times[0,1]) \cap L_{l o c}^{2}\left(\left[0, \infty\left[; W_{0}^{1,2}(0,1)\right)\right.\right.\right.\right.$ vérifie $(6)$, alors pour presque tout $T>0$, on a

$$
\iint_{(0, T) \times(0,1)} v_{x}^{2}+\int j\left(b(v(T, .)) \leq \int j\left(b\left(v_{0}\right)\right)\right.
$$


où $j$ est la fonction convexe conjuguée de la primitive de $b$ nulle en 0 ; et donc, supposant que $v$ satisfait (7), on a

$$
\iint v_{x}^{2} \leq\left\|v_{0}\right\|_{\infty}\left\|b\left(v_{0}\right)\right\|_{1}
$$

Notons maintenant qu'il suffit de prouver le théorème pour les données initiales $v_{0}$ vérifiant

$$
v_{0} \in W_{0}^{1,2}(0,1), \quad v_{0}^{\prime} \in B V(0,1),
$$

où $B V(0,1)$ est l'espace des fonctions à variation bornée sur $(0,1)$.

Supposons en effet que le théorème soit vrai pour de telles données initiales ; donnons-nous $v_{0} \in L^{\infty}(0,1)$ et une suite $\left(f_{n}\right)$ de $W^{2,1}(0,1)$ convergeant p.p. sur $(0,1)$ vers $v_{0} ;$ définissons pour tout $n, m \in \mathbb{N}$

$$
v_{0, n, m}=\inf \left(\left\|v_{0}^{+}\right\|_{\infty}, \sup \left(-\left\|v_{0}^{-}\right\|_{\infty}, f_{n}, f_{n+1}, \ldots, f_{n+m}\right)\right) .
$$

L'espace $\left\{f \in W_{0}^{1,2}(0,1) ; f^{\prime} \in B V(0,1)\right\}$ est réticulé puisque $\left(f^{+}\right)^{\prime}=f^{\prime} \chi_{\{f>0\}} ;$ donc les données initiales $v_{0, n, m}$ vérifient (15); notons $v_{n, m}$ les solutions correspondantes. Utilisant (9) et (7), la suite $\left(v_{n, m}\right)$ est décroissante en $n$, croissante en $m$ et bornée par $\left\|v_{0}\right\|_{\infty}$; posons $v=\lim _{n \rightarrow \infty} \lim _{m \rightarrow \infty} v_{n, m}$. Il est clair que $v$ vérifie (7); d'après (14), la suite $\left(v_{n, m}\right)$ est bornée dans $L^{2}\left(0, \infty ; W_{0}^{1,2}(0,1)\right)$ et $v$ vérifie $(14)$ à la limite. Passant à la limite dans (6) appliquée à $v_{n, m}$, on voit que $v$ est solution correspondant à $v_{0}$.

Soit $\underline{v}_{0} \in L^{\infty}(0,1)$ avec $\underline{v}_{0} \geq v_{0}$ p.p. $\operatorname{sur}(0,1)$; on peut l'approcher par une suite $\left(\underline{f}_{n}\right)$ de $W^{2,1}(0,1)$ de telle sorte que $\underline{f}_{n} \geq f_{n}$ pour tout $n$; construisant $\underline{v}_{0, n, m}, \underline{v}_{n, m}, \underline{v}$ correspondants, on aura $\underline{v}_{0, n, m} \geq v_{0, n, m}$, d'où $\underline{v}_{n, m} \geq v_{n, m}$ par utilisation de (9), et donc $\underline{v} \geq v$ à la limite. Notant enfin que la solution $v$ ne dépend que de $b\left(v_{0}\right)$ d'après l'unicité prouvée ci-dessus, et que si $b\left(\underline{v}_{0}\right) \geq b\left(v_{0}\right)$, on peut toujours trouver $\hat{v}_{0}, \underline{\hat{v}}_{0} \in L^{\infty}(0,1)$ tels que $\underline{\hat{v}}_{0} \geq \hat{v}_{0}, b\left(\hat{v}_{0}\right)=b\left(v_{0}\right)$ et $b\left(\underline{\hat{v}}_{0}\right)=b\left(\underline{v}_{0}\right)$, on voit que (9) est satisfaite pour toutes données initiales dans $L^{\infty}(0,1)$.

Nous allons en fait montrer le résultat suivant :

Proposition. Sous les hypothèses du théorème, pour tout v vérifiant (15), il existe une solution (forte) v satisfaisant

$$
\begin{aligned}
v \in L^{\infty}\left(0, \infty ; W^{2,1}(0,1) \cap W_{0}^{1,2}(0,1)\right), & b(v) \in W^{1, \infty}\left(0, \infty ; L^{1}(0,1)\right), \\
b(v)_{t}=v_{x x}+F(v)_{x} & \text { p.p. } \operatorname{sur}(0, \infty) \times(0,1), \\
b(v)(0, \cdot)=b\left(v_{0}\right) & \text { p.p. } \operatorname{sur}(0,1) .
\end{aligned}
$$

Il est clair qu'une telle solution forte est solution de (6). D'autre part, si $\underline{v}$ est solution de (16) correspondant à une autre donnée initiale $\underline{v}_{0}$ vérifiant $(15)$ et $b\left(\underline{v}_{0}\right) \geq b\left(v_{0}\right)$, on aura $b(\underline{v}) \geq b(v)$ d'après $(8)$; donc pour p.p. $t \in(0, \infty)$, on aura $(v(t)-\underline{v}(t))_{x x}+(F(v(t))-F(\underline{v}(t)))_{x}=0$ p.p. sur l'ouvert $\{v(t)>\underline{v}(t)\}$ de $(0,1)$; raisonnant comme dans la preuve du Lemme 1 , on voit que $\{v(t)>\underline{v}(t)\}=\emptyset$ pour p.p. $t \in(0, \infty)$, c'est à dire que (9) est satisfaite. De la même manière, on voit qu'une solution forte de (16) vérifie (7).

Pour achever de prouver le théorème, il suffit donc de montrer la proposition. À cette fin introduisons l'opérateur (multivoque) $A$ de $L^{1}(0,1)$ défini par

$$
A u=\left\{-\left(v^{\prime \prime}+F(v)^{\prime}\right) ; v \in W^{2,1}(0,1) \cap W_{0}^{1,1}(0,1), u=b(v) \text { p.p. sur }(0,1)\right\} .
$$


Remarquons que d'après le Lemme 1 , étant donné $f \in A u$, il existe un unique $v \in W^{2,1}(0,1) \cap W_{0}^{1,1}(0,1)$ tel que $u=b(v), f=-\left(v^{\prime \prime}+F(v)^{\prime}\right)$ p.p. sur $(0,1)$. Il en résulte qu'étant donné $v_{0}$ vérifiant $(15)$, il existe une solution $v$ de (16) si et seulement si il existe une solution $u$ de

$$
u \in W^{1, \infty}\left(0, \infty ; L^{1}(0,1)\right), u^{\prime}(t)+A u(t) \ni 0 \text { p.p. } t \in(0, \infty), u(0)=b\left(v_{0}\right) .
$$

En effet, si $v$ est solution de (16), il est clair que $u=b(v)$ est solution de (18) ; réciproquement, si $u$ est solution de $(18)$, la fonction $v:(0, \infty) \rightarrow W^{2,1}(0,1) \cap W_{0}^{1,1}(0,1)$ définie p.p. $t \in(0, \infty)$, grâce au Lemme 1 , par

$$
v(t)^{\prime \prime}+F(v(t))^{\prime}=u^{\prime}(t) \quad \text { p.p. sur }(0,1)
$$

sera solution de (16).

On a le résultat suivant :

Lemme 2. L'opérateur $A$ de $L^{1}(0,1)$ défini par (17) est T-accrétif, m-accrétif et inversible.

Preuve. Ce lemme s'obtient facilement à partir de la Proposition 2.4 de [3]. Pour être complet donnons rapidement une preuve directe. Puisque $f \in A u$ si et seulement si $u=b(v)$ avec $v \in W^{2,1}(0,1) \cap W_{0}^{1,1}(0,1)$ solution de $-\left(v^{\prime \prime}+F(v)^{\prime}\right)=f$ p.p. sur $(0,1)$, par application du Lemme 1 , l'opérateur $A^{-1}$ est une application croissante continue et compacte de $L^{1}(0,1)$ dans $\mathcal{C}_{0}(0,1)$.

Montrons la T-accrétivité de $A:$ pour $i=1,2$, considérons $f_{i} \in A u_{i}$ et soit $v_{i} \in W^{2,1}(0,1) \cap W_{0}^{1,1}(0,1)$ tel que $u_{i}=b\left(v_{i}\right), f_{i}=-\left(v_{i}^{\prime \prime}+F\left(v_{i}\right)^{\prime}\right)$; posons $u=u_{1}-u_{2} f=f_{1}-f_{2}, v=v_{1}-v_{2}$. Si $] \alpha, \beta[$ est une composante de l'ouvert $\{x \in] 0,1[; v(x)>0\}$, on a $v(\alpha)=v(\beta)=0, v^{\prime}(\beta) \leq 0 \leq v^{\prime}(\alpha)$ et donc $\int_{] \alpha, \beta[} f \geq 0$; d'où $\int_{\{v>0\}} f \geq 0$. On en déduit pour tout $\lambda>0: \int u^{+}=\int_{\{v>0\}} u \leq \int_{\{v>0\}}(u+\lambda f) \leq \int(u+\lambda f)^{+}$.

Montrons la m-accrétivité de $A$ : fixons $f \in L^{1}(0,1)$. L'application $G(\lambda, u)=A^{-1}(f-\lambda u)$ est continue compacte de $[0,1] \times L^{1}(0,1)$ dans $\mathcal{C}_{0}(0,1)$; d'après l'accrétivité de $A$, l'ensemble $\left\{(\lambda, u) \in[0,1] \times L^{1}(0,1) ; G(\lambda, u)=\right.$ $u$ \} est borné ; donc, d'après le théorème de Schauder, il existe $u \in L^{1}(0,1)$ tel que $G(1, u)=u$, c'est-à-dire $u+A u \ni f$.

Preuve de la Proposition. L'opérateur $A$ étant m-accrétif, on peut appliquer la théorie des semi-groupes non linéaires $\left(c f\right.$. [4] ou [2]). Fixons $v_{0}$ satisfaisant (15). Pour $\epsilon>0$ donnons-nous $v_{\epsilon}^{0} \in W^{2,1}(0,1) \cap W_{0}^{1,1}(0,1)$ tel que

$$
\left\|b\left(v_{\epsilon}^{0}\right)-b\left(v_{0}\right)\right\|_{1} \leq \epsilon,\left\|v_{\epsilon}^{0^{\prime \prime}}\right\|_{1} \leq \operatorname{var}\left(v_{0}^{\prime}\right)
$$

et définissons $u_{\epsilon}:\left[0, \infty\left[\rightarrow L^{1}(0,1)\right.\right.$ par :

$$
u_{\epsilon} \text { est linéaire sur }[n \epsilon,(n+1) \epsilon], u_{\epsilon}(n \epsilon)=(I+\epsilon A)^{-n} b\left(v_{\epsilon}^{0}\right) \text { pour tout } n \in \mathbb{N} \text {. }
$$

Alors $(c f .[4]) u_{\epsilon} \rightarrow u$ dans $\mathcal{C}\left(\left[0, \infty\left[; L^{1}(0,1)\right)\right.\right.$ lorsque $\epsilon \rightarrow 0$. De plus, puisque $f_{\epsilon}=-\left(v_{\epsilon}^{0^{\prime \prime}}+F\left(v_{\epsilon}^{0}\right)^{\prime}\right) \in A b\left(v_{\epsilon}^{0}\right)$ est bornée dans $L^{1}(0,1)$, les fonctions $u_{\epsilon}$ sont bornées dans $W^{1, \infty}\left(0, \infty ; L^{1}(0,1)\right)$ et la fonction $u$ est lipschitzienne de $\left[0, \infty\left[\right.\right.$ dans $L^{1}(0,1)$. Nous allons montrer que $u$ est en fait dans $W_{\text {loc }}^{1,1}\left(0, \infty ; L^{1}(0,1)\right)$. Utilisant la théorie abstraite $(c f .[4])$, il en résultera que $u$ vérifie (18) et la proposition sera démontrée.

Pour prouver que $u \in W_{\text {loc }}^{1,1}\left(0, \infty ; L^{1}(0,1)\right)$, notons que $u_{\epsilon}(n \epsilon)=b\left(v_{\epsilon}^{n}\right)$ où $v_{\epsilon}^{n}$ est défini par

$$
b\left(v_{\epsilon}^{n}\right)=\epsilon\left(v_{\epsilon}^{n \prime \prime}+F\left(v_{\epsilon}^{n}\right)^{\prime}\right)+b\left(v_{\epsilon}^{n-1}\right) \text { pour } n \in \mathbb{N} .
$$


Par accrétivité $\left\|v_{\epsilon}^{n \prime \prime}+F\left(v_{\epsilon}^{n}\right)^{\prime}\right\|_{1} \leq\left\|v_{\epsilon}^{0^{\prime \prime}}+F\left(v_{\epsilon}^{0}\right)^{\prime}\right\|_{1}$ est bornée et donc, d'après (12), $\left\|v_{\epsilon}^{n^{\prime}}\right\|_{\infty}$ est bornée. Il en résulte, par continuité de $b$, que les fonctions $u_{\epsilon}^{n}$ sont équicontinues sur $[0,1]$ et donc, d'après le théorème d'Ascoli, que $u \in \mathcal{C}\left(\left[0, \infty[\times[0,1])\right.\right.$ et $u_{\epsilon} \rightarrow u$ uniformément sur tout compact de $[0, \infty[\times[0,1]$.

Posons $\Omega=\{(t, x) \in] 0, \infty[\times] 0,1[; u(t, x) \in \mathbb{R} \backslash K\}$; c'est un ouvert de $Q=] 0, \infty[\times] 0,1[$. Puisque $u$ est lipschitzienne de $\left[0, \infty\left[\right.\right.$ dans $L^{1}(0,1), u_{t}$ est une mesure de Radon sur $Q$ et $K$ étant négligeable par hypothèse, on a $u_{t}=u_{t} \chi_{\Omega}$.

Considérons un rectangle $\omega=] t_{0}-\delta, t_{1}+\delta[\times] x_{0}-\delta, x_{1}+\delta[$ relativement compact dans $\Omega$; il existe un compact $\kappa$ de $\mathbb{R} \backslash K$ tel que pour tout $\epsilon>0$ suffisamment petit, $u_{\epsilon}(\omega) \subset \kappa$. Donnons-nous $\xi \in \mathcal{D}(] x_{0}-\delta, x_{1}+\delta[)$ tel que $0 \leq \xi \leq 1, \xi=1$ sur $\left[x_{0}, x_{1}\right]$. On a pour $t_{0}-\delta \leq(n-1) \epsilon<t<n \epsilon \leq t_{1}+\delta$,

$$
\begin{gathered}
c_{0} \int_{x_{0}}^{x_{1}}\left(\frac{\partial u^{\epsilon}}{\partial t}(t)\right)^{2} \leq c_{0} \int_{0}^{1} \xi^{2}\left(\frac{u_{\epsilon}^{n}-u_{\epsilon}^{n-1}}{\epsilon}\right)^{2} \leq \int_{0}^{1} \xi^{2} \frac{u_{\epsilon}^{n}-u_{\epsilon}^{n-1}}{\epsilon} \frac{v_{\epsilon}^{n}-v_{\epsilon}^{n-1}}{\epsilon} \\
=-\int_{0}^{1} \xi^{2} v_{\epsilon}^{n \prime} \frac{v_{\epsilon}^{n^{\prime}}-v_{\epsilon}^{n-1^{\prime}}}{\epsilon}+\int_{0}^{1}\left(2 \xi \xi^{\prime}+\xi^{2} F^{\prime}\left(v_{\epsilon}^{n}\right)\right) v_{\epsilon}^{n \prime} \frac{v_{\epsilon}^{n}-v_{\epsilon}^{n-1}}{\epsilon} \\
\leq \frac{1}{\epsilon} \int_{0}^{1} \frac{\xi^{2}}{2}\left(v_{\epsilon}^{n-1^{\prime 2}}-v_{\epsilon}^{n \prime^{2}}\right)+c_{1}\left(\int_{0}^{1} \xi^{2}\left(\frac{u \epsilon^{n}-u_{\epsilon}^{n-1}}{\epsilon}\right)^{2}\right)^{1 / 2}\left\|2 \xi^{\prime}+\xi F^{\prime}\left(v_{\epsilon}^{n}\right)\right\|_{\infty}\left\|v_{\epsilon}^{n \prime}\right\|_{\infty},
\end{gathered}
$$

avec $c_{0}=\left(\max _{b^{-1}(\kappa)} b^{\prime}\right)^{-1}, c_{1}=\left(\min _{b^{-1}(\kappa)} b^{\prime}\right)^{-1}$.

Donc il existe une constante $C$ telle que

$$
\iint_{](n-1) \epsilon, n \epsilon[\times] x_{0}, x_{1}\right]}\left(\frac{\partial u^{\epsilon}}{\partial t}(t)\right)^{2} d x \leq C\left(\epsilon+\int_{0}^{1} \frac{\xi^{2}}{2}\left(v_{\epsilon}^{n-1^{\prime 2}}-v_{\epsilon}^{n \prime^{2}}\right)\right) .
$$

En sommant pour les $n$ tels que $t_{0}-\delta \leq(n-1) \epsilon<n \epsilon \leq t_{1}+\delta$, on voit que $\frac{\partial u_{\epsilon}}{\partial t}$ reste borné dans $L^{2}(] t_{0}, t_{1}[\times] x_{0}, x_{1}[)$. A la limite, $\frac{\partial u}{\partial t} \in L^{2}(] t_{0}, t_{1}[\times] x_{0}, x_{1}[)$. Ceci étant vrai pour tout rectangle $\omega$ relativement compact dans $\Omega$, on a $\frac{\partial u}{\partial t} \in L_{\text {loc }}^{2}(\Omega)$ et donc $u_{t}=u_{t} \chi_{\Omega} \in L^{\infty}\left(0, \infty ; L^{1}(0,1)\right)$.

\section{Remarques}

La même technique peut s'appliquer à des équations plus générales de la forme (1) en dimension 1 d'espace ; on peut aussi évidemment considérer d'autres conditions limites que celles de Dirichlet. Par contre, restant dans le cas d'une équation de la forme (4), nous ignorons si l'on peut relaxer considérablement l'hypothèse sur la fonction $b$. En effet la technique repose sur l'existence de solutions fortes pour des données initiales suffisament régulières, et même dans le cas $F=0$, ceci n'est plus vrai pour des fonctions $b$ continues croissantes quelconques.

L'extension à la dimension d'espace supérieure à 1 pose des problèmes de capacité ; elle sera considérée ultérieurement.

D'après les résultats de [3], la fonction $b(v)$ est continue dans $C\left(\left[0, \infty\left[; L^{1}(0,1)\right)\right.\right.$ par rapport aux données $v_{0}$, $b$ et $F$. Par contre nous ignorons s'il y a continuité de la fonction $v$ elle-même. Dans cet ordre de problème, nous ignorons même si la fonction $v_{\epsilon}$ considérée dans la preuve de la Proposition converge (faiblement) vers la solution $v$. 


\section{REFERENCES}

[1] H.W. Alt and S. Luckhaus, Quasi-linear elliptic-parabolic differential equations. Math. Z. 183 (1983) 311-341.

[2] Ph. Bénilan, M.G. Crandall and A. Pazy, Evolution equations governed by accretive operators. Book in preparation.

[3] Ph. Bénilan and P. Wittbold, On mild and weak solutions of elliptic-parabolic problems. Adv. Diff. Equ. 1 (1996) 1053-1073.

[4] M.G. Crandall and T. Liggett, Generation of semigroups of nonlinear transformations on general Banach spaces. Amer. J. Math. 93 (1971) 265-298. 